\title{
Investigation of tautomeric structures of thionin by satellite holes: matrix dependence
}

\author{
Kevin C. Weng a,1, Chien-Chih Chiang a,b Ji-Yen Cheng a,b,2, Shao-Ying Cheng a, \\ Roman I. Personov ${ }^{\mathrm{c}}$, Ta-Chau Chang ${ }^{\mathrm{a}, *}$ \\ a Institute of Atomic and Molecular Sciences, Academia Sinica, P.O. Box 23-166, Taipei 10764, Taiwan \\ b Department of Chemistry, National Taiwan University, Taipei 10764, Taiwan \\ ${ }^{\mathrm{c}}$ Institute of Spectroscopy, Academy of Sciences of Russia, Troitsk, Moscow Region 142092, Russian Federation
}

Received 23 October 1998; in final form 5 January 1999

\begin{abstract}
We have applied the satellite hole spectroscopy to study the tautomeric structures of thionin dye in glycerol:water glass. Slightly different frequencies $\left(\sim 15 \mathrm{~cm}^{-1}\right)$ are observed for several satellite holes of thionin upon tuning the burning wavelength, implying that two conformational structures exist. However, only one set of satellite holes occurring in the spectrum of thionin doped in polyvinyl butyral film allows us to investigate the tautomeric structures of thionin in different matrices. Our results suggest that the appearance of two tautomeric structures of thionin in glycerol:water glass is due to the proton exchange between different sites of thionin and the matrix through intermolecular hydrogen bond. (C) 1999 Elsevier Science B.V. All rights reserved.
\end{abstract}

\section{Introduction}

The excited-state vibrational spectrum obtained by the hole burning method was first reported by two groups $[1,2]$. When laser excitation is tuned to the region of vibronic transitions in the inhomogeneously broadened absorption spectrum, a number of satellite holes resulting from Franck-Condon (FC) active vibrational modes can be produced in the hole

\footnotetext{
* Corresponding author. Fax: +8862 2362 0200; e-mail: tcchang@po.iams.sinica.edu.tw

${ }^{1}$ Present address: Department of Material Science, Stanford University.

${ }^{2}$ Present address: Institute of Biomedical Sciences, Academia Sinica.
}

burned spectrum. The distances between laser line and such satellite holes provide frequencies of the molecular vibrations in excited state. Until recently, the advantages of satellite hole ( $\mathrm{SH}$ ) spectroscopic method [3,4] and the application of $\mathrm{SHs}$ to the monitoring of change in local interactions have been emphasized [5-8]. The difference between pre- and post-burn absorption spectra can give a backgroundfree SH spectrum. More importantly, when tuning the laser excitation to the vibronic transition, laser excites several types of centres which have their vibronic transitions at the laser wavelength. Therefore, each SH can be viewed as an individual electronic transition of its own vibronic transition. As a result, the intensity of $\mathrm{SH}$ can be enhanced by the inverse FC factor [3]. The SH spectrum is particularly useful for providing the low-frequency modes 
$\left(<500 \mathrm{~cm}^{-1}\right)$, which are difficult to be obtained in the infrared and Raman spectra. In addition, the SHs resulting from combination modes are useful for determining the structural geometry [3]. Furthermore, the low quantum yield photoproduct can be accumulated and revealed by the antiholes in the hole burned spectrum [4]. These advantages allow us to investigate the mechanism of photoinduced reaction of 9-aminoacridine (9AA) doped in glycerol:water $\left(\mathrm{Gl}: \mathrm{H}_{2} \mathrm{O}\right)$ glasses at different $\mathrm{pH}$ values $[3,4]$. In addition, this SH spectral method is very useful to probe chromophore-DNA interactions, specifically the binding modes of chromophores to the DNA and their conformational structures [5-8].

The phenothiozinium dyes, such as thionin and methylene blue, have been studied for their photodamaging effects upon binding to DNA [9-12]. It was believed that the binding mode of a photosensitizer can influence the photoreaction mechanism [13]. Thus, a comprehensive study of the vibrational modes is essential to identify the binding of the chromophore to DNA substrates for better understanding the photochemical reaction. Recently, Marek et al. [14] suggested that there are two possible sites in the thionin molecule that can bind the first proton, either the $\mathrm{NH}$ group or the $\mathrm{N}$ atom of the central ring. These two structures are shown in Scheme 1. To our knowledge, no experimental evidence has been reported. In this work, we have applied the SH method to prove the proposed structures of thionin by the two sets of SHs as a function of burning wavelength. These two sets of SHs are independent of $\mathrm{pH}$ values between 2 and 12 and the concentration of $1 \times 10^{-4}$ to $2 \times 10^{-3} \mathrm{M}$ of thionin in $\mathrm{Gl}: \mathrm{H}_{2} \mathrm{O}$ glasses. We have further performed the $\mathrm{SH}$ spectrum of methylene blue for comparison since there is no such b-form for methylene blue. In addition, the SH spectrum of thionin doped in polyvinyl butyral (PVB) film provides more interesting results. These results provide the insight of the matrix dependence for the tautomeric structures of thionin.

\section{Experimental}

The experimental setup for the $\mathrm{SH}$ spectrum has been described elsewhere [15]. Holes were produced by using a sync-pumped dye laser pumped by a mode-locked and Q-switched Nd:YAG laser. The dye laser emitted trains of $\sim 10$ pulses separated by $13 \mathrm{~ns}$ at a repetition rate of $500 \mathrm{~Hz}$. The FWHM of the dye laser was $\sim 2 \mathrm{~cm}^{-1}$. The absorption spectrum was obtained by dispersing the output of a xenon arc lamp (Oriel 66083) through a double-beam spectrometer with a resolution of $\sim 0.03 \mathrm{~nm}$. The spot size of the burning beam was $\sim 1 \mathrm{~mm}$ and that of the reading beam was $\sim 200 \mu \mathrm{m}$.

Thionin was purchased from Sigma and used after HPLC purification. Methylene blue was also purchased from Sigma and used without purification. Dye molecule was dissolved in $\mathrm{Gl}_{2} \mathrm{H}_{2} \mathrm{O}(5: 4, \mathrm{v} / \mathrm{v})$ solution and then injected into a quartz tube with an inner diameter of $1.5 \mathrm{~mm}$ and placed in a brass holder with several drill holes of $\sim 1 \mathrm{~mm}$ diameter. Clear glasses were formed by introducing the sample directly into a Janis dewar from room temperature to $6 \mathrm{~K}$. The sample of thionin in PVB film was prepared by dissolving the thionin and the PVB powder (Polysciences) in organic solvent of $\mathrm{CH}_{2} \mathrm{Cl}_{2}$. The syrupy solutions were poured onto a glass plate and placed into a desiccator to dry into a film of thickness $\sim 70 \mu \mathrm{m}$.<smiles></smiles>

a-form<smiles></smiles>

b-form

Scheme 1. Thionin structures. 


\section{Results and discussion}

\subsection{Tautomeric structures of thionin}

Fig. 1a,b shows the pre- and post-burn spectra of thionin doped in $\mathrm{Gl}: \mathrm{H}_{2} \mathrm{O}$ glass taken at $\lambda_{\mathrm{B}} \sim 575$ and $590 \mathrm{~nm}$, respectively. The difference between each set of spectra gives the $\mathrm{SH}$ spectrum as shown in Fig. 1c,d. Fig. 1 shows two intriguing features. First, a broad antihole located around $603 \mathrm{~nm}$ is independent of the burning wavelength, implying that the hole formation is mainly due to photochemical process and this antihole is a photoproduct of the photoreaction. The most intriguing feature is the slight frequency-shift from 798 to $809 \mathrm{~cm}^{-1}$ and 955 to $975 \mathrm{~cm}^{-1}$ when $\lambda_{\mathbf{B}}$ is tuned from 575 to $590 \mathrm{~nm}$. In addition, the $429 \mathrm{~cm}^{-1}$ peak disappears, but the $308 \mathrm{~cm}^{-1}$ peak appears in Fig. 1d. In order to make a better comparison of the two sets of SHs, Fig. 2 shows more $\mathrm{SH}$ spectra of thionin taken at $\lambda_{\mathrm{B}}$ $\sim 565,580,595$, and $605 \mathrm{~nm}$ at $6 \mathrm{~K}$. Besides the changes of certain SHs observed in Fig. 1, it is shown in Fig. 2 that additional SHs at 478, 1140, and $1275 \mathrm{~cm}^{-1}$ are blue-shifted to 489,1152 , and $1297 \mathrm{~cm}^{-1}$, respectively. The two sets of excitedstate vibrational frequencies suggest that at least two conformational structures of thionin have appeared in $\mathrm{Gl}: \mathrm{H}_{2} \mathrm{O}$ glass.

The absorption spectra at $\sim 6 \mathrm{~K}$ and room temperature are shown in the inset of Fig. 2. Our absorption spectrum of thionin in $\mathrm{Gl}: \mathrm{H}_{2} \mathrm{O}$ at room temperature is red-shifted by $\sim 5 \mathrm{~nm}$ compared to the absorption spectrum of thionin in water [16]. This $\sim 5 \mathrm{~nm}$ difference may be due to different solvation shells. At present, it is not clear why the $\sim 617 \mathrm{~nm}$ band is not distinct in the room temperature spectrum. Nevertheless, the two absorption bands at 604 and $\sim 617 \mathrm{~nm}$ in the low-temperature spectrum may be due to the two conformational structures.

We now consider the possible structures of thionin existing in $\mathrm{Gl}: \mathrm{H}_{2} \mathrm{O}$ glass. Considering the aggregation of thionin molecules [16] and the various protonations of thionin [14], we have conducted concentration-dependent $\left(1 \times 10^{-4}\right.$ to $\left.2 \times 10^{-3} \mathrm{M}\right)$ and

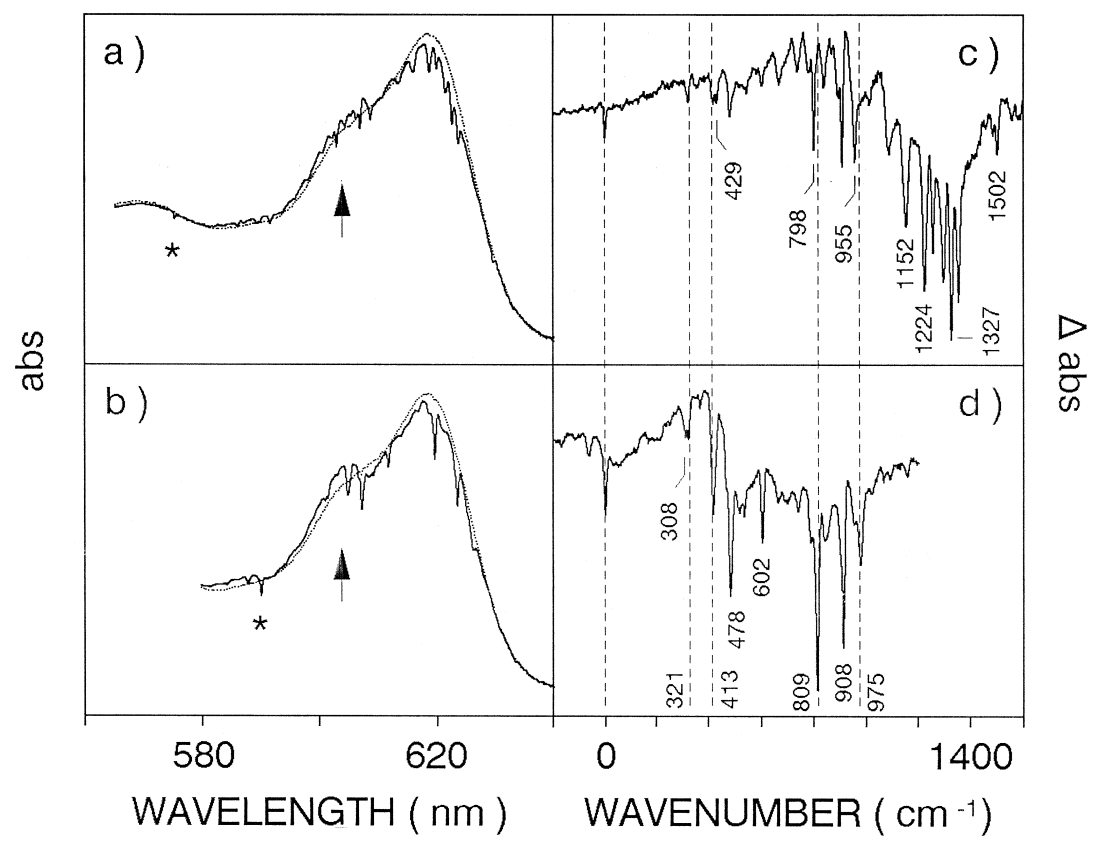

Fig. 1. Pre- (doted line) and post-burn (solid line) spectra of thionin doped in $\mathrm{Gl}_{2} \mathrm{H}_{2} \mathrm{O}$ glass taken at $\lambda_{\mathrm{B}} \sim$ (a) $575 \mathrm{~nm}$ and (b) $590 \mathrm{~nm}$, and their corresponding SH spectra (c) and (d) at $6 \mathrm{~K}$. The pulse energy is $\sim 1.0 \mu \mathrm{J}$ with burn time of 10 min for each spectrum. The asterisk (*) denotes the burning position. The arrow shows the antihole. Prominent satellite holes are labeled with excited-state vibrational frequencies. The deviation of the labeling frequency is $\pm 3 \mathrm{~cm}^{-1}$. 


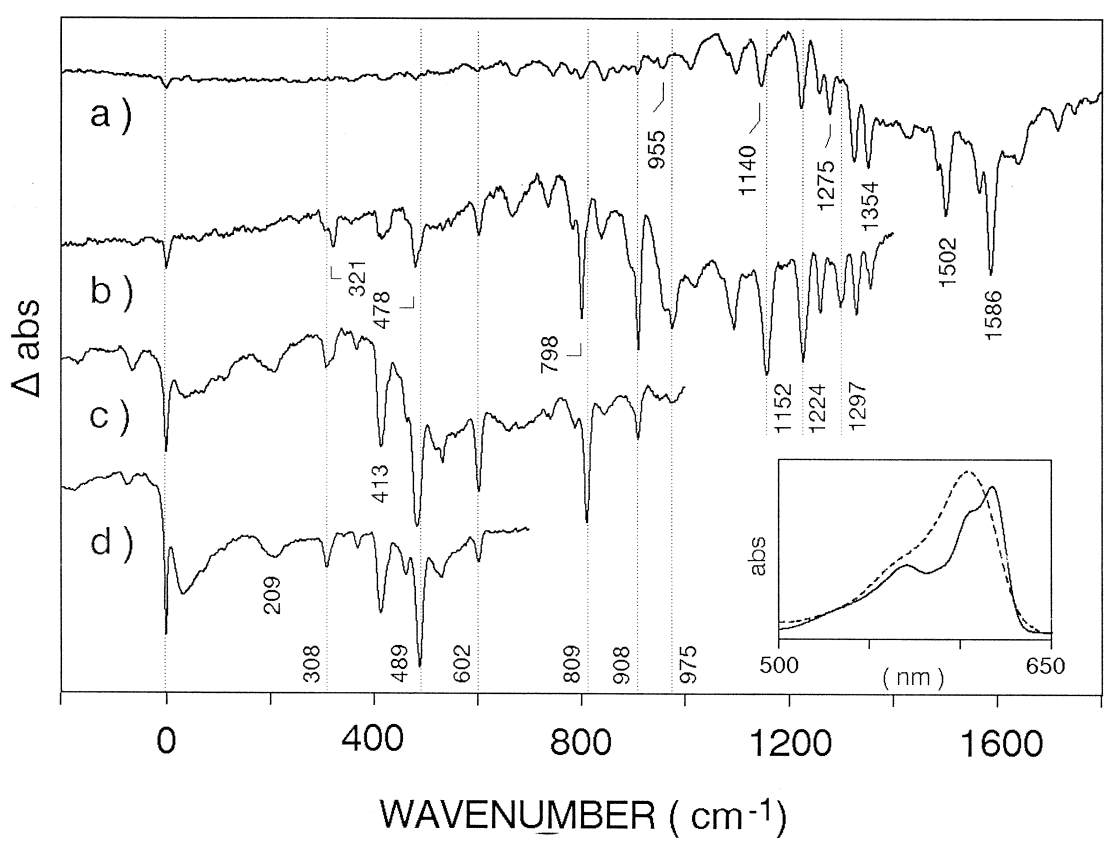

Fig. 2. Satellite hole spectra of thionin doped in Gl: $\mathrm{H}_{2} \mathrm{O}$ glass taken at $\lambda_{\mathrm{B}} \sim$ (a) $565 \mathrm{~nm}$, (b) $580 \mathrm{~nm}$, (c) $595 \mathrm{~nm}$ and (d) $605 \mathrm{~nm}$ at $6 \mathrm{~K}$. Their corresponding pulse energies are $\sim 1.2, \sim 1.0, \sim 0.8$, and $\sim 0.5 \mu \mathrm{J}$ with the same burn time of 10 min for each spectrum. The inset shows the absorption spectra taken at room temperature (dash line) and $6 \mathrm{~K}$ (solid line).

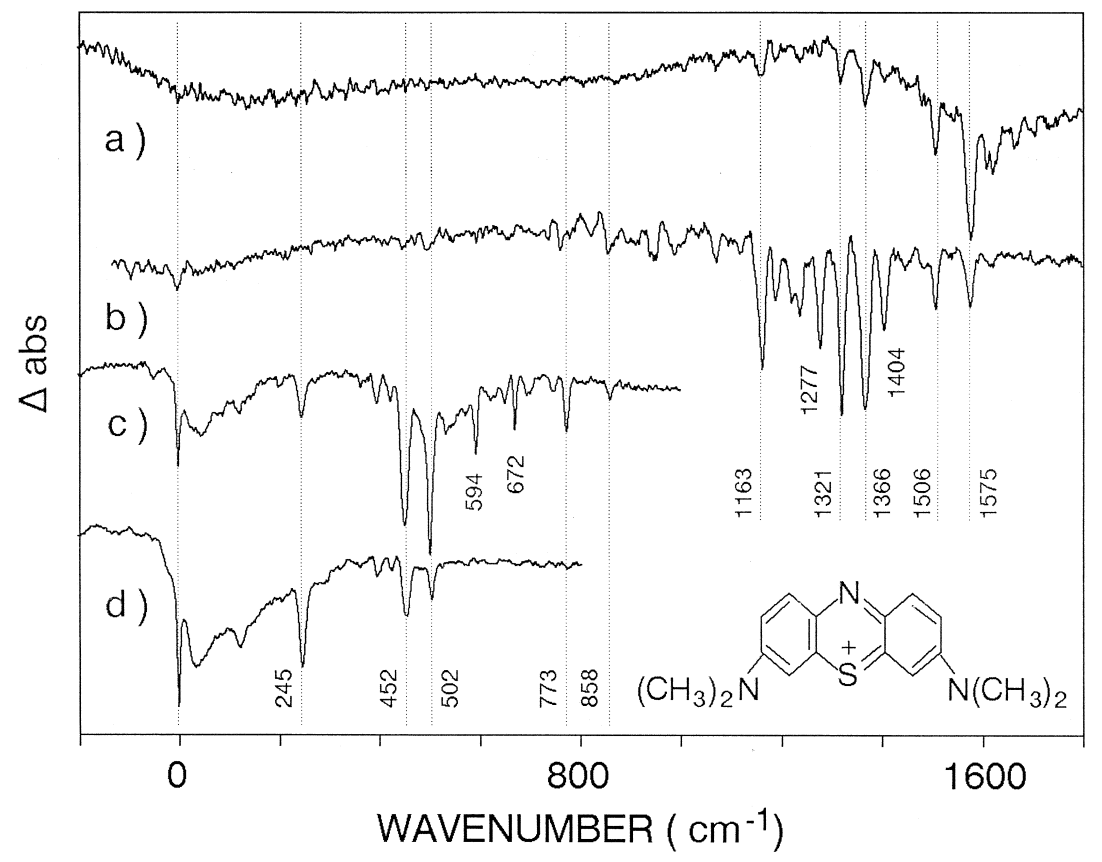

Fig. 3. Satellite hole spectra of methylene blue doped in Gl: $\mathrm{H}_{2} \mathrm{O}$ glass taken at $\lambda_{\mathrm{B}} \sim$ (a) 610 , (b) 625 , (c) 655 and (d) $670 \mathrm{~nm}$ at $6 \mathrm{~K}$. The pulse energies are $\sim 0.4, \sim 0.2, \sim 0.2$, and $\sim 0.1 \mu \mathrm{J}$ with the burn times of $20,10,10$ and $20 \mathrm{~min}$, respectively. The chemical structure of methylene blue is given in the figure. 
$\mathrm{pH}$-dependent $(2,7$, and 12) $\mathrm{SH}$ spectra to examine whether the counterpart of SHs is due to either thionin dimer or thionin-proton complexes. The two sets of SHs are observed for both cases (data not shown), indicating that the counterpart of SHs is not due to either thionin dimer or different degrees of protonation. It is noted that the color of thionin disappears in more basic solution.

Therefore, we suggest that the two sets of excited vibrational frequencies are due to two tautomers as described in Scheme 1. This conjecture can be tested by methylene blue since there is no such b-form described in Scheme 1 for methylene blue. Fig. 3 shows SH spectra of methylene blue doped in $\mathrm{Gl}: \mathrm{H}_{2} \mathrm{O}$ glass as a function of the burning wavelength at $\sim 6 \mathrm{~K}$. Only one set of the SHs is observed for methylene blue, implying that the two sets of vibrational frequencies of thionin in $\mathrm{Gl}: \mathrm{H}_{2} \mathrm{O}$ glass are most likely due to the presence of two tautomeric structures as shown in Scheme 1.

\subsection{Normal mode calculations and mode coupling}

Normal mode calculations on the a-form of thionin for the qualitative assignment of the SHs were conducted in vacuo by using a Hyperchem package. The conformational geometry of thionin is optimized by the Polak-Ribiere algorithm and then applied to perform PM3 semi-empirical calculations. Restricted Hartree-Fock wave functions are used. Fig. 4 shows the calculated diagrams of the $311,424,501,629$, 836 , and $1020 \mathrm{~cm}^{-1}$ modes of thionin. The 501 and $836 \mathrm{~cm}^{-1}$ modes are mainly due to inner ring motions and the 311 and $629 \mathrm{~cm}^{-1}$ modes are related to ring skeleton motions. The 424 and $1020 \mathrm{~cm}^{-1}$ modes involve $\mathrm{NH}_{2}$ motions.

Careful examination of the frequencies of SHs in Fig. 2 suggests that the $321 \mathrm{~cm}^{-1} \mathrm{SH}$ can be due to the difference between either the 798 and $478 \mathrm{~cm}^{-1}$ SHs or the 809 and $489 \mathrm{~cm}^{-1}$ SHs. In addition, the $1275 \mathrm{~cm}^{-1} \mathrm{SH}$ can be assigned to the sum of the 798 and $478 \mathrm{~cm}^{-1} \mathrm{SHs}$ and the $1297 \mathrm{~cm}^{-1} \mathrm{SH}$ to the sum of 809 and $489 \mathrm{~cm}^{-1}$ SHs. Furthermore, the $955 \mathrm{~cm}^{-1} \mathrm{SH}$ is the overtone of the $478 \mathrm{~cm}^{-1} \mathrm{SH}$ and the $975 \mathrm{~cm}^{-1} \mathrm{SH}$ is the overtone of the 489 $\mathrm{cm}^{-1} \mathrm{SH}$. We believe that the $(478,798) \mathrm{cm}^{-1}$ and $(489,809) \mathrm{cm}^{-1} \mathrm{SHs}$ are related to the inner ring motions of the two tautomers of thionin.

\subsection{Mechanism of antihole and matrix dependence}

We now turn our attention to the formation of antihole. In the previous study of the 9AA doped in $\mathrm{Gl}: \mathrm{H}_{2} \mathrm{O}$ glasses [3], the coincidence between the

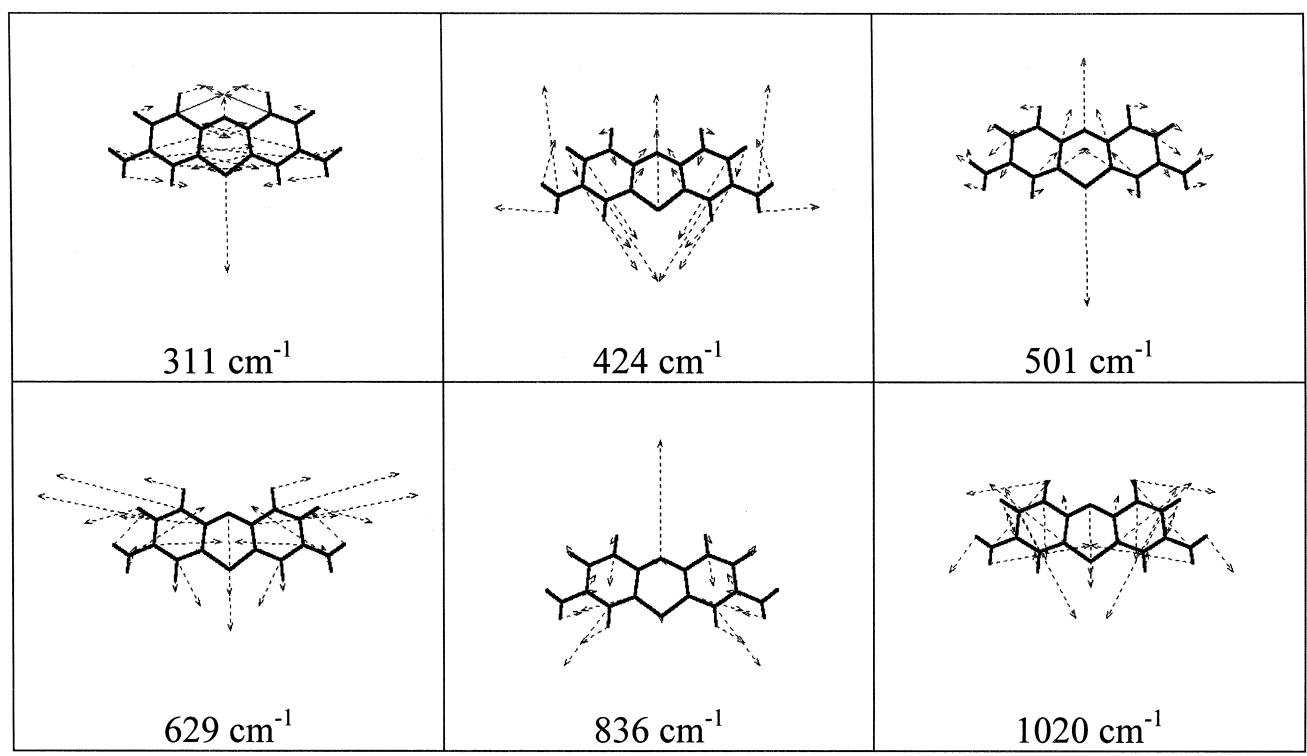

Fig. 4. Normal mode calculations of six ground-state symmetric vibrational frequencies of thionin. 
antiholes in the spectrum of the imino-form of neutral 9AA and the absorption bands of monoprotonated 9AA suggested that the monoprotonated 9AA is the photoproduct of the imino-form of neutral 9AA. In addition, we proposed that proton transfer between chromophore and matrix is the mechanism for the photoreaction. In this work, the broadly superimposed hole at $\sim 617 \mathrm{~nm}$ and the broad antihole at $\sim 603 \mathrm{~nm}$ imply that the component corresponding to the $\sim 603 \mathrm{~nm}$ band can be the photoproduct of the component corresponding to the $\sim 617 \mathrm{~nm}$ band. We further anticipate that the antihole appeared in $\mathrm{Gl}: \mathrm{H}_{2} \mathrm{O}$ glass may be due to proton exchange between different sites of thionin and matrix via intermolecular hydrogen bonding.

In order to test our conjecture of the antihole formation of thionin in $\mathrm{Gl}: \mathrm{H}_{2} \mathrm{O}$ glass, Fig. 5 shows $\mathrm{SH}$ spectra of thionin doped in PVB film as a function of the burning wavelength. This is because no acidic proton is provided by PVB. The absorption spectra of thionin in PVB film at $\sim 6 \mathrm{~K}$ and room temperature are shown in the inset of Fig. 5. The lowest absorption band is observed at $\sim 608 \mathrm{~nm}$ in both spectra and no additional band is found at low-temperature absorption spectrum. In addition, we do not observe the broad antihole around $603 \mathrm{~nm}$ in the spectrum of thionin doped in PVB film. The most striking feature is that the SHs at 489, 809, 975, 1152, and $1297 \mathrm{~cm}^{-1}$ in Fig. 2 disappear in Fig. 5. The presence of the 320 and $430 \mathrm{~cm}^{-1}$ SHs in Fig. 5 can be assigned to the difference between the 798 and $478 \mathrm{~cm}^{-1}$ SHs and the difference between the 907 and $478 \mathrm{~cm}^{-1} \mathrm{SHs}$, respectively.

The relative narrow absorption band and the lack of the counterpart of SHs in the spectra of thionin in PVB film suggest that one of the proposed tautomers of thionin does not exist in PVB film. It is noted that the thionin is not dissolved in $\mathrm{CH}_{2} \mathrm{Cl}_{2}$ without adding PVB powder. PM3 calculations give dipole moments of $1.76 \mathrm{D}$ for the a-form and $4.97 \mathrm{D}$ for the $\mathrm{b}$-form of thionin. Since the dipole moment of the b-form is much larger than that of the a-form, we suggest that the a-form of thionin is the main structure in PVB film. In the study of 9AA [3], we found more SHs corresponding to fundamental modes of the aminoform of monoprotonated 9AA than the imino-form

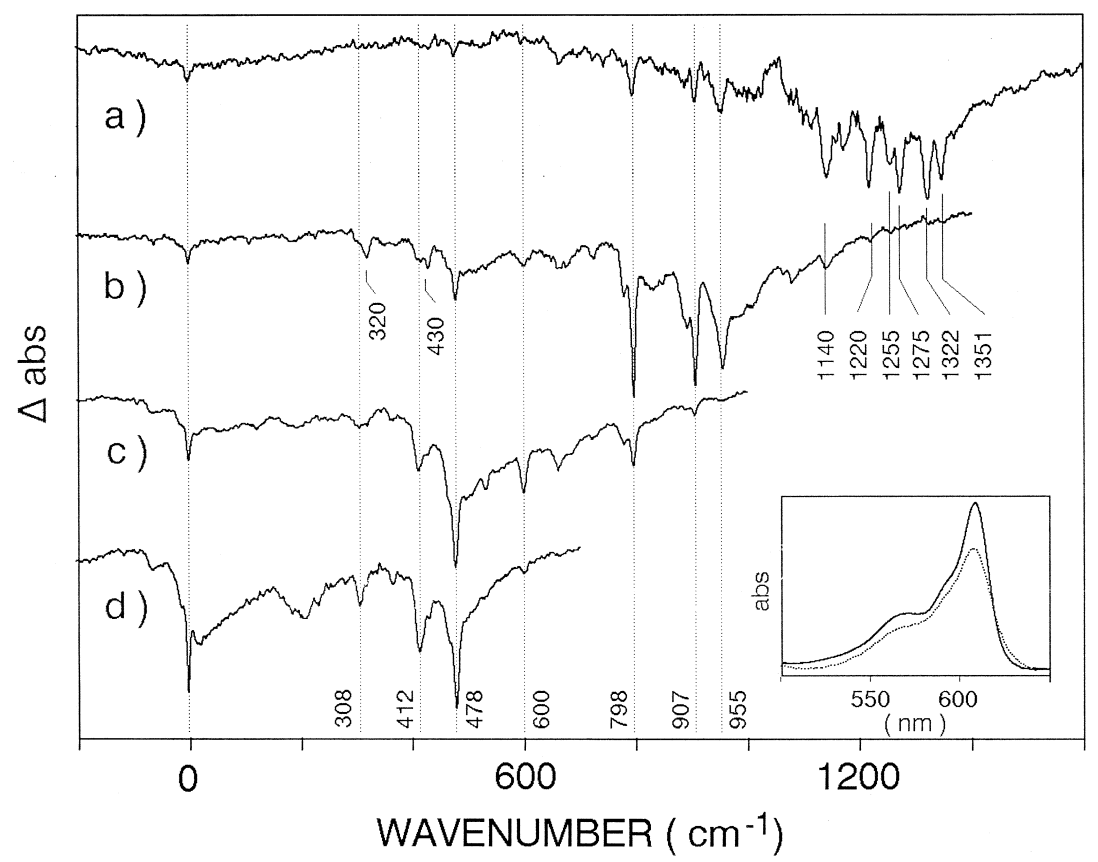

Fig. 5. Satellite hole spectra of thionin doped in PVB film taken at $\lambda_{\mathrm{B}} \sim$ (a) 570, (b) 580, (c) 590 and (d) $600 \mathrm{~nm}$ at $6 \mathrm{~K}$. The pulse energies are $\sim 1.0 \mu \mathrm{J}$ for spectra $(\mathrm{a}-\mathrm{c})$ and $\sim 0.5 \mu \mathrm{J}$ for spectrum $(\mathrm{d})$ with the same burn time of 30 min for each spectrum. The inset shows the absorption spectra taken at room temperature (dash line) and $6 \mathrm{~K}$ (solid line). 
of neutral 9AA. This is because a symmetric mode generally has a large FC factor. The presence of the fundamental modes at 308, 412, 600 and $907 \mathrm{~cm}^{-1}$ SHs along with the 478 and $798 \mathrm{~cm}^{-1}$ SHs in Fig. 5 also suggests that the a-form of thionin is more likely the structure in PVB film. Thus, we can assign the $501 \mathrm{~cm}^{-1}$ mode to the $478 \mathrm{~cm}^{-1} \mathrm{SH}$ and the $836 \mathrm{~cm}^{-1}$ mode to the $798 \mathrm{~cm}^{-1} \mathrm{SH}$.

The two sets of $(478,798) \mathrm{cm}^{-1}$ and $(489,809)$ $\mathrm{cm}^{-1}$ SHs can be used to identify the a-form and b-form of thionin, respectively. Since the 489 and $809 \mathrm{~cm}^{-1}$ SHs are produced while tuning $\lambda_{\mathrm{B}}$ to the long wavelength, the band around $603 \mathrm{~nm}$ is ascribed to the a-form and the red-side of the $617 \mathrm{~nm}$ band to the b-form of thionin in $\mathrm{Gl}: \mathrm{H}_{2} \mathrm{O}$ glass.

Our results suggest that both two tautomeric forms of thionin are simultaneously presented in $\mathrm{Gl}: \mathrm{H}_{2} \mathrm{O}$ glass and only the a-form of thionin appears in PVB film. The main difference between $\mathrm{Gl}: \mathrm{H}_{2} \mathrm{O}$ glass and PVB film is the presence and absence of acidic proton in the matrix, respectively. Thus, we speculate that the occurrence of these two tautomeric forms in $\mathrm{Gl}: \mathrm{H}_{2} \mathrm{O}$ glass can be closely related to intermolecular hydrogen bonds formed between the $\mathrm{OH}$ group of the matrix to the $\mathrm{NH}$ group and the $\mathrm{N}$ atom of the central ring of the thionin. Considering the excitation of both forms of thionin in $\mathrm{Gl}: \mathrm{H}_{2} \mathrm{O}$ glass, the proton exchange between these two types of intermolecular hydrogen bonding is very possible responsible for the antihole formation. The appearance of the antihole around $603 \mathrm{~nm}$ suggests that the a-form of thionin in $\mathrm{Gl}: \mathrm{H}_{2} \mathrm{O}$ glass is more produced than the b-form after photoinduced reaction. On the other hand, the intermolecular hydrogen bonding formed between the thionin and the PVB is very unlikely. As a result, only the relative stable form of thionin appears in PVB film. Our results suggest that the acidic proton provided by matrix can be very critical for molecule which has various binding sites for the formation of hydrogen bonding.

\section{Conclusion}

In summary, we have demonstrated that the siteselectivity of the $\mathrm{SH}$ spectrum provides a very powerful method for distinguishing different species with similar absorption bands. In this work, we report experimental results that the two tautomeric structures of thionin proposed by Marek et al. [14] exist in $\mathrm{Gl}: \mathrm{H}_{2} \mathrm{O}$ glass. However, the presence of these tautomers is matrix-dependent. The acidic proton from matrix assists the appearance of the two tautomers. The identification of the two tautomers by their specific SHs is very important for the study of the interactions of thionin with DNA in buffer solution, which is under progress in our laboratory.

\section{Acknowledgements}

This work was supported by the Academia Sinica and the NSC of the Republic of China (Grant No. NSC-87-2113-M001-016). RIP appreciates the NSC from Taiwan to support his visit to IAMS for 2 months.

\section{References}

[1] A.A. Gorokhovskii, J. Kikas, Opt. Commun. 21 (1977) 272.

[2] B.M. Kharlamov, L.A. Bykovskaya, R.I. Personov, Chem. Phys. Lett. 50 (1977) 407.

[3] C.C. Chiang, B.C. Hwang, J. Yu, J.Y. Cheng, C.Y. Mou, S.H. Lin, T.-C. Chang, J. Chem. Soc. Faraday Trans. 93 (1997) 1297.

[4] C.C. Chiang, C.Y. Mou, T.-C. Chang, Chem. Phys. Lett. 273 (1997) 153

[5] J.Y. Cheng, C.C. Chiang, Y.R. Cheng, H.S. Chen, L.J. Lin, T.-C. Chang, J. Chin. Chem. Soc. 44 (1997) 97.

[6] J.Y. Cheng, S.H. Lin, T.-C. Chang, J. Phys. Chem. B 102 (1998) 5542.

[7] C.T. Kuo, C.C. Chiang, J. Yu, K. Peck, T.-C. Chang, J. Chem. Soc. Faraday Trans. 94 (1998) 1989.

[8] T.-C. Chang, C.T. Kuo, C.C. Chiang, J.Y. Cheng, C.S. Yan, K. Peck, submitted.

[9] E. Tuite, J.M. Kelly, J. Photochem. Photobiol. B: Biol. 21 (1993) 103

[10] M. Kubista, T. Hard, P.E. Nielsen, B. Norden, Biochemistry 24 (1985) 6336.

[11] P. Hagmar, S. Pierrou, P. Nielsen, B. Norden, M. Kubista, J. Biomol. Struct. Dyn. 9 (1992) 667.

[12] E.M. Hogan, J. LeGrange, B. Austin, Nature 304 (1983) 752.

[13] E. Tuite, J.M. Kelly, Biopolymers 35 (1995) 419.

[14] N. Marek, S. Kunsági-Máté, K. Szabó, J. Mol. Struc. (THEOCHEM) 315 (1994) 187.

[15] T.-C. Chang, C.C. Chiang, G.J. Small, S.H. Chou, Chem. Phys. Lett. 223 (1994) 190.

[16] W.C. Lai, N.S. Dixit, R.A. Mackay, J. Phys. Chem. 88 (1984) 5364. 